\title{
The lessons to take home
}

\author{
N A MITCHISON \\ University College London, Department of Zoology, Gower Street, \\ London, UK
}

The message from this meeting can be divided into four parts concerning, firstly molecular biology, secondly immunodiagnosis, thirdly vaccine development, and fourthly immunopathology.

As regards the molecular biology the first message is to echo Enger's point that the introduction of these monoclonal antibodies for screening and other purposes, has indeed been a triumph of co-operation. It is a valuable achievement of the WHO Tropical Disease Research programme.

The second major message is that the proteins that have been picked out of Richard Young's recombinant DNA library form a famous five, the 12, 18, 28, 36 and $65 \mathrm{KD}$ molecules. Yet time after time during this meeting we have learned that these comprise no more than a tiny sample of the antigens of this mycobacterium. For example the crossed electrophoresis pictures of Morten Harboe's showed a multitude of antigens. For each of those antigens there are no doubt helper T cells that are driving the antibody response. Why don't they figure among the famous five? And again the biochemical studies of Wheeler told of a whole cascade of enzymes. Why have not those figured as antigens?

A key point about this paradox came from de Vries' paper showing that around $80 \%$ of the $T$ cell reactivity in nearly 20 clones isolated from infected humans recognizes just this narrow part of the spectrum. Before we accept that this is the window through which we need to look at the immunology of mycobacteria, it is clear that further effort is needed to open that window.

I agree, but only up to a point, with Tore Godal that limiting dilution examination of $\mathrm{T}$ cells is appropriate. The techique has definite limitations. There are three things which one wants to know about T cells. One is the full potential repertoire. Another is the fraction of cells that will actually react under in vivo conditions, and a third is the fraction clonable with a particular procedure selected by the investigator. Those three fractions are by no means necessarily equal to one another. My guess is that the fraction which is clonable can be very strongly manipulated, and the work in Freiburg clearly demonstrates this.

I a m sceptical about the notion of clonal expansion among $\mathrm{T}$ cells as judged from limiting dilution data. It seems to me that much of the data which is interpreted as clonal expansion, may simply represent activation without expansion so that cells then score in the limiting dilution assay. And even when T cells do expand clonally they seem to hit a plateau after just a few days under in vivo conditions. Clonal expansion in the T cell population may occur, but it is under very much tighter homeostatic control than is the case for B cells.

How will that window be opened in the future? We heard during the course of this meeting of some plans. Dr. Mustafa is initiating a blind T cell screen, and my colleague Padmini Salgame has also been doing just that. 
Jonathan Lamb's methodology for using electrophoresis and immunoblotting looks like a nice way of opening the window. We heard Anders on the use of antisera as distinct from monoclonal antibodies for screening. The ultimate goal is a plate of $E$.coli clones expressing the Young library, on which a $\mathrm{T}$ cell carpet will be spread, so that we shall be able to pick reactive $\mathrm{T}$ cells in the same way that we can pick antibodies just now.

A last point about molecular biology is that the work on carbohydrates has been a triumph. We must be impressed by the PGL-I disaccharide synthesis, by the construction of conjugates, and also by the work on LAM. It is doubtful whether increasing the size of the carbohydrate unit beyond the disaccharide will add to specificity, although studies which have been made with blood groups and also with dextrans indicate that the affinity of the antigen-antibody reaction may increase with size up to about 5 saccharides. But whether that will help form complexes between multivalent antibody and antigens is doubtful.

Immunodiagnosis is making steady progress, as we heard from Drs. Abe on salivary IgA, and from Drs. Kaldany and Engers on urinary immunodiagnosis using the PGL-I antigen.

The future of immunodiagnosis according to Shinnick, Kim, Buchanan and Young lies in refinement of epitope analysis, refinement which will be based in part on peptide synthesis and in part on recombinant DNA. At first sight the aim of this work is to find the perfect epitope that will discriminate between different mycobacterial species. However, historically specificity is a property not of a single species of antibody, but of the sets present in antisera, and there are good theoretical reasons for thinking that a single discriminatory epitope may be an inappropriate aim.

In addition, the smaller the structure which is recognized, the more tightly defined the epitope, the larger the amount of antibody which will be needed. So, the less sensitive the test becomes. I am sure that Dr. Buchanan would agree that we are not really looking for a single epitope but for a set of epitopes. The larger that set is, outside the $65 \mathrm{KD}$ protein if necessary, the better it will be.

We heard intriguing suggestions that the $\mathrm{T}$ epitopes which are recognized may reflect the condition of infection. For example vaccinated individuals recognized preferentially the 18 $\mathrm{KD}$ protein, whereas naturally immunized individuals prefer the 36 and $65 \mathrm{KD}$ proteins.

As regards B cells Dr. Patarroyo gave ample evidence that in tuberculosis the spectrum of antibodies changes. Clearly this spectrum of reactivity needs examination in field tests. We heard from Dr. Mustafa that as little as $25 \mathrm{ml}$ of blood would suffice for those tests, although he did tell us that the $\mathrm{T}$ cells would need expansion with a possible bias introduced by the expanision procedure.

Why do we need improved immunodiagnosis? Well, Dr. Gill raised the possibility that within a vaccinated group we may be able to pick out individuals who become infected. By appropriate serology we might detect antibodies against antigens which generate a response in infection but not after vaccination. It would be an enormous help in the WHO trials if infection could to detected on this basis.

More widely, immunodiagnosis is needed for early detection of the disease, and also to tell us when the disease is cured so that chemotherapy can be stopped. It is clear that immunodiagnosis in this sense is worthwhile if, and only if, it becomes a criterion on which chemotherapy is either instituted or discontinued. Dr. Abe told us that the clinicians take little notice of serology at present. We need not to be too worried about that. The history of the development of diagnostic tests, at least in the first world, is that they take a long time to be validated. The TDR programme of WHO has an important part to play in the introduction and validation of immunodiagnostic tests. 
On the vaccine front we heard the encouraging figure from Dr. Fine, and repeated by Dr. Pönnighaus, of more than $50 \%$ protection produced by BCG vaccination under certain conditions and in certain areas. This surely strengthens the case for a true Mycobacterium leprae-based vaccine.

Dr. Convit discussed the therapeutic use of vaccines. We heard about the dangers of reversal reactions, a problem of immunological intervention in the established disease, and this certainly adds a cautionary note.

How about the second generation vaccines based on protein tayloring and recombinant DNA that were discussed by Bloom? The important point here is that they are likely to have significant advantages apart from economy and safety. In particular, they can be expected to be more effective because (i) destractor or smoke-screen epitopes of the type discussed by Anders can be deleted, (ii) suppressor epitopes can be deleted, and (iii) agretopes and their associated T-epitopes can be optimally located.

A word about the anti-idiotype approach. I agree with Barry Bloom that this still needs adequate testing. It came as a surprise to learn that the anti-idiotype antibody could elicit $\mathrm{T}$ cells able to react with the original antigen. For instance in Ivanyi's case to react with the 36 KD antigen would not have been expected because of the tremendous difference between the antibody combining site and the T cell receptor. But similar findings have been made by Ertl in Boston so the question now is whether responses that are worthwhile for vaccination purposes can indeed be generated in this way.

Fourthly, immunopathology. About what have we agreed?

First there is general agreement, notably from Kaufmann and from Kaplan, about what may be called the post-Mackaness theory of protective immunity. That is that $\mathrm{T}$ cells activate macrophages through lymphokines, of which as Kaplan emphasized the principal and only clearly identified one is gamma interferon. But there are also indications of other macrophage activating factors, such as lymphotoxin and also other unidentified proteins secreted by $\mathrm{T}$ cells.

There is less agreement about the role of cytotoxic cells. Kaufmann and J. Louis both indicated that cytotoxic cells play a part in the defence mechanism. They both did so on the basis of rather short terms systems, for instance by Kaufmann after challenge at day 10 in a newly reconstituted mouse. So it remains to be seen whether the kind of long term effects which Lagrange studied also involve cytotoxic $\mathrm{T}$ cells. If they do, then there is a clear prediction that Class I Ir genes should be involved. So far there is no sign of that in either mouse or man. That is my main reason for scepticism about the role of cytotoxic $\mathrm{T}$ cells.

It is agreed, in tissues at least, that lepromatous as distinct from tuberculoid leprosy is characterized by a reduced frequency of $\mathrm{T} 4$ positive cells and an increase in $\mathrm{T} 8$ cells.

It is clear that this is accompanied by specific suppression, as Reitan re-emphasized in her studies. It is clear also that the lepromatous individuals can and do make large amounts of antibody, much of which is probably, although not certainly, T cell dependent so they must have activated $T$ cells somewhere. The dissenting voice in all this was Dr. Nath who finds that in blood T4 cells are present but functionally inhibited by monocytes. Thorsby raised a very pertinent question about the specificity of that inhibition, and they agreed that further work is needed before those monocytes can be judged guilty of manifesting a specific inhibitory effect.

What is the nature of the specific suppressor mechanism which operates in lepromatous leprosy?

The genetics of lepromatous leprosy tell us that Class II molecules guide the choice between lepromatous and tuberculoid leprosy. We are already familiar with that from the work of the Leiden group in co-operation with Dr. Convit in Venezuela. Family studies indicate 
dominant control of lepromatous leprosy, perhaps through suppression mediated by Class II HLA genes. Chinese studies, again from Dr. de Vries' work from Leiden, confirm the importance of Class II molecules and map those molecules to the DQ sublocus. That is the same story which Sasazuki is now telling us, again that Class II molecules control suppressor cells, and that DQ is the key control element. The DQ molecules presumably control the activity of T4 suppressor-inducer cells, which in turn control the activity of T8 suppressor-effectors.

What are these immune suppressor (Is) genes doing in the human population at all?

Immune response (Ir) genes are easy to understand. They protect us against acute infection, most notably against viruses. But why should we have immune suppression genes if they are bad for us? I would like to suggest that immunopathology should not be regarded as a passive phenomenon, something which simply happens as a concomitant of immunity, but that it may play an evolutionarily more dynamic role. According to this view the main source of tissue damage is not hypersensitivity in the sense of autoimmunity, or hypersensitivity to environmental antigens, but is rather chronic infection that generates immunopathology in the way we understand it in the context of tuberculoid leprosy. This selects for immune suppression genes that reduce tissue damage. This evolutionary pathway constitutes a feed back loop in which parasites generate immunopathology in order to select for immune suppression genes. The immunopathology is a deterrent which the parasite uses to select for immune suppressor genes in the host. They in turn damp down the immune response against the parasite and thus permit the parasite to live longer. So, immune suppressor epitopes and immune suppressor genes from this point of view can be regarded as an evolutionary collusion between the parasite and its host. 